\title{
PERCEPCIÓN DE LAS NIIF EN LOS CONTADORES HÁBILES EN EL COLEGIO DE CONTADORES DE AREQUIPA
}

\author{
PERCEPTION OF IFRS ON BUSINESS COUNTERS \\ IN AREQUIPA ASSOCIATION OF ACCOUNTANTS
}

Abel Marvin Arce Arenas y Mirza Yasmin Quintana Simon

Universidad Católica San Pablo, Arequipa, Perú

\section{Resumen:}

Esta investigación describe el grado de conocimiento y preferencia en cuanto la aplicación de las normas tributarias en la elaboración de la información financiera que la utilización de las Normas Internacionales de Información Financiera (NIIF), por parte de los contadores varones y mujeres hábiles en el Colegio de Contadores de nuestra ciudad; para lo cual se ha tomado una muestra probabilística de 50 contadores entre varones y mujeres. Dentro de los resultados se tiene que el $66 \%$ de encuestados no aplica las NIIF, además que el $86 \%$ de la muestra respondió las preguntas de conocimiento básico sobre NIIF de forma incorrecta, y que la mayoría de los encuestados prefiere la aplicación de las normas tributarias por sobre las NIIF en la elaboración de la información con propósito general.

Palabras clave: Percepción de normas, NIIF, contadores.

\section{Summary:}

This research describes the level of awareness and preference for the application of tax regulations in the preparation of the financial information that the use of International Financial Reporting Standards (IFRS) by accountants men and women who have the qualification of working in the Association of Accountants from Arequipa; for which it has taken a random sample of 50 accountants. Among the findings that were obtained, $66 \%$ of respondents did not apply IFRS, also $86 \%$ of the sample answered questions 
about basic knowledge of IFRS incorrectly, and the majority of accountants prefer the application of the tax rules over IFRS, in the preparation of information with general purpose.

Keywords: Perception of standards, IFRS, accountants.

\section{Introducción}

El concepto de globalización se apoya en una serie de aspectos fundamentales como son "la interdependencia económica y la integración física de los mercados, la estandarización de productos, la homogeneización de las demandas nacionales y la visión de que las ventajas competitivas no se alcanzan por la suma de los países sino por la integración de actividades coordinadas a nivel global" (Arroyo, 2011, p. 68). Además, como dijo Paul Pacter-ex miembro IASB-, desde 1975 hasta este momento en que se ha producido la globalización de los mercados del mundo, los inversionista buscan invertir en cualquier parte del mundo al menor costo posible (Mantilla, 2013).

Como consecuencia de la globalización, el contexto financiero exige el uso de estándares internacionales que homogenicen los procedimientos contables en las empresas. En relación a ello, el International Accounting Standards Board (IASB) (2014) indica que de 130 perfiles jurisdiccionales (que representan más del $96 \%$ de la capitalización total en el mundo) en el 81\% ya es obligatorio el uso de las NIIF para todas o la mayoría de las empresas que cotizan en la bolsa, mientras que la mayoría de las otras jurisdicciones permiten las NIIF. Esto se debe a desde hace algunos años, hay esfuerzos importantes que han redundado en la mejora de la comparabilidad de la información financiera de todas las empresas que actúan en un mismo mercado globalizado (Palacios \& Martínez, 2005).

Nuestro país también está inserto en las políticas liberales afines a la globalización de los mercados, lo cual se evidencia en el crecimiento sostenido que ha venido teniendo el país, en los últimos años. Por ejemplo, el crecimiento del PBI en el 2014 será mayor al del 2013 en 5,7\%; además que Standard E Poor's (S\&P) elevó la calificación soberana de Perú a BBB+ debido a la fortaleza de nuestra economía. Por otro lado, los estudios hechos por Ernst $\mathcal{E}$ Young (2014) nos indican que en el 2013 nuestra ciudad creció $10.2 \%$ y se espera para el 2014 un crecimiento mayor al 13\%. También se informó que el $52 \%$ del PBI se genera en las regiones fuera de Lima, y el sur, gracias a un fuerte dinamismo en la actividad empresarial Arequipa está a la cabeza. Por último, el 2020 se espera que nuestra Región Arequipa logre una inversión privada aproximada de USD 8,ooo millones (Ministerio de Economía y Finanzas, 2014).

\section{Necesidad de las NIIF}

Las NIIF (principios contables) son emitidas por el IASB, organismo internacional, cuyo objetivo es optimizar la información contable, haciéndola oportuna, real, relevante y comparable (a nivel mundial y con periodos anteriores) para la adecuada toma de decisiones por los usuario (clien- 
tes, proveedores, accionistas, el gobierno, etc.). En nuestro país las hemos adoptado y oficializado por el Consejo Normativo de Contabilidad, ente que indica en sus resoluciones que el contador peruano está obligado a conocerlas y aplicarlas (Lam, 2010).

Para el año 2013, las empresas que tuvieron ingresos mayores a 30,00o UITs y cotizan en bolsa, debieron presentar sus estados financieros auditados y acorde a las NIIF ante la SMV. Para este año, solo las empresas obligadas a presentar esta información son las que tienen ingresos mayores a 15,00o UIT. Se espera que las empresas que tengan 3,00o UIT de ingresos publiquen su información financiera para el año 2015; en pocas palabras, se quiere que progresivamente todas las empresas utilicen NIIF para optimizar la información contable.

Por otro lado, nuestro país está creciendo económicamente y de manera regular: para este año se estima un crecimiento de $5 \%$, que es muy atractivo para la inversión extranjera y nacional. Este crecimiento también se observa en nuestra ciudad, siendo el hito comercial para invertir en todo el sur; por ello han llegado este año empresas extranjeras de España y Alemania para invertir en el sector construcción, trayendo millones de euros para invertir. Además, Ernst $\mathcal{E}$ Young una de las empresas más poderosas en brindar servicios de auditoría, impuestos, transacciones y consultoría a nivel mundial llega a nuestra ciudad en marzo del 2014, con profesionales de alta calidad provenientes de la ciudad de Lima, para brindar sus servicios de auditoría de acuerdo a las NIIF. Por ello, Valle (2013) informa que las NIIF constituyen parte de los datos complejos manejados actualmente en el mundo de los negocios, cuyo cabal entendimiento, interpretación y aplicación constituye dominio de pocos y cotizados especialistas.

Por el contrario, el contador arequipeño en general es "sunatista" y "tributarizado", tiene la necesidad de capacitarse en NIIF, pero en nuestra ciudad hay poca difusión de este tema, en comparación con Lima (Alvarado, 2012). En ese sentido el Colegio de Contadores de Lima organizó el XVII Diplomando de NIIF entre mediados del año pasado y el presente, mientras que el Colegio de Contadores de Arequipa solo se hizo un PEP de NIIF y varios cursos de tributación. Es más, el IASB (2014) a nivel mundial está emitiendo nuevas NIIF, la 14 y la 15, y con posterioridad las NIC serán remplazadas por las NIIF. En consecuencia hay la preocupación que los contadores arequipeños se capaciten en NIIF e razón del crecimiento económico y las exigencias mundiales.

\section{Proceso de adopción de las NIIF}

El International Accounting Standards Committee (IASC) se formalizó a través de un acuerdo entre los organismos representativos de la profesión contable de: Australia, Canadá, Francia, Alemania, Japón, México, Holanda, Reino Unido e Irlanda y los Estados Unidos de Norteamérica en 1973 (Díaz, Gil \& Vilchez, 2010). Además, Zans (2009) nos aclara que el IASC emitió las NIC (IAS por sus siglas en ingles) y SIC (no se usó la traducción, se les llamo SIC en los países hispanos). Estas NIC emitidas por el IASC, tienen por objetivo, formular normas contables para la profesión y promover su 
aceptación y adopción internacional, así como propender hacia la armonización de regulaciones y procedimientos relativos a la preparación y presentación de estados financieros (Díaz et al, 2010).

En el año 2001 el IASC fue remplazado por el IASB, organismo que adoptó las NIC y emitió las NIIF. En un futuro las NIC desaparecerán, debido a que cuando se emite una NIIF tiende a suprimir las NIC.

Al respecto Saavedra (2012) nos manifiesta que todo este tiempo ha existido una situación muy variada en el ámbito de la contabilidad por parte de los distintos países del mundo, pues no todos han adoptado las NIIF. Zans (2010) por su parte, nos informa que nuestro CNC es el encargado de oficializar las NIIF, y que el contador está obligado a aplicarlas las NIIF oficializadas.

Además nuestro Plan Contable General Empresarial (PCGE) ha sido homogenizado con las NIIF, contemplando aspectos relacionados con la preparación y revelación de la información (Zans, 2012). En ese sentido, según Hirache y Gonzales (2011) nuestro CNC ha elaborado el Nuevo Plan Contable Empresarial (PCGE), que ha sido una reingeniería de los conocimientos contables, debido a que a partir del año 2011 empieza su aplicación, y este PCGE se encuentra homogenizado con las NIIF.

En importante señalar que entre las normas nacionales aplicables a la NIIF tenemos La ley General de Sociedades (Ley No. 26887) que en su artículo 223, señala que las NIC adquieren reconocimiento legal, al indicarse que los estados financieros deben elaborarse de acuerdo a disposiciones legales sobre la materia y con Principios de Contabilidad Generalmente Aceptados (PCGA) en el país (Díaz, 2010, p. 14). Posteriormente el CNC, mediante la resolución 013-98-EF/93.01, precisó los alcances de los principios de contabilidad generalmente aceptados que comprenden las NIC.

Por otro lado, según Ley $\mathrm{N}^{\circ}$ 29782, la CONASEV se trasforma en la Superentendía de Mercados y Valores (SMV). Este ente vela por el adecuado manejo de las empresas y por la contabilidad de las mismas, por ello se encarga de emitir resoluciones para un mejor control contable. Al respecto, el 2011 se publica la Ley $\mathrm{N}^{\circ} 29720$ que establece que las empresas con ingresos mayores 3,00o UITs deben presentar sus ingresos auditados de acuerdo con las NIIF (Díaz, Durán y Valencia, 2012). Sin embargo, hubo modificaciones a las resoluciones y las empresas obligadas a presentar la información financiera serían: para el ejercicio del 2013, aquellas que tengan ingresos mayores a 30,00o UITs, mientras que para este año, aquellas empresas que tengan ingresos mayores a 15,00o UITs, y para el 2015 aquellas que tengas más de 3,00o UITs.

\section{Metodología}

\section{Muestra}

Esta investigación se llevó a cabo bajo la perspectiva del muestreo no probabilístico. La muestra está constituida por los contadores públicos colegiados del Colegio de Contadores Públicos de la ciudad de Arequipa, que para esta investigación constaba de 50 personas. 


\section{Instrumentos}

Utilizamos el cuestionario elaborado para medir las variables en la recolección de datos usando preguntas de tipo cerrado de carácter dicotómico, semi-cerradas y politómicas en una escala de tipo Likert.

\section{Procedimientos}

Esta investigación la desarrollamos a través de una encuesta con un instrumento que fue auto-administrado en las instalaciones del Colegio Público de Contadores de la ciudad de Arequipa.

\section{Resultados}

A continuación se procederá a detallar el análisis de las preguntas del cuestionario. Las preguntas referidas a la situación de los contadores, nos indican que de los 50 encuestados, 33 trabajan como dependientes en una empresa, siete trabajan como independientes en un estudio contable y 10 trabajan como dependientes e independientes.

De los 50 encuestados, 26 contadores trabajan en empresas comerciales, 18 trabajan en empresas industriales y seis trabajan en servicios. Del total de los 50 encuestados, 28 trabajan en una mediana empresa, dos en una empresa grande y 20 en pequeñas y micro empresas.

Por otro lado, el promedio de los que están ejerciendo la profesión es 12 años. Asimismo, del total de los encuestados, solo 5 contadores han recibido capacitación en las NIIF en el 2012, 15 en el 2013 y 19 en el 2014.
Sobre las preguntas referidas al conocimiento básico y preferencia por el uso de las NIIF, de los 50 encuestados, 3 de ellos indicaron que no saben qué son las NIIF. De los 47 que indicaron conocer las NIIF, 17 de indicaron que sí aplican las NIIF en la elaboración de su información financiera. Dentro de este grupo, siete respondieron las preguntas de conocimiento básico de forma correcta.

Tabla 1.

Conocimiento de las NIIF.

\begin{tabular}{|c|c|c|c|}
\hline & CONOCEN & \multicolumn{2}{|c|}{ APLICAN } \\
\hline NIIF & & SI & NO \\
\hline SI & 47 & 17 & 30 \\
\hline NO & 3 & 0 & 3 \\
\hline Total general & $\mathbf{5 0}$ & $\mathbf{1 7}$ & 33 \\
\hline
\end{tabular}

Del total de encuestados, 13 personas respondieron bien la pregunta "Cuando se detectan errores en la presentación de los EEFF de periodos anteriores", de los cuales siete son menores de 35 años y seis son mayores de 35 años. De los que han respondido bien esta pregunta solo cinco personas son los que conocen y aplican NIIF, siendo el 90\% mujeres.

Sobre la pregunta referida a la depreciación que se aplica en contrato de leasing, la establecida en las normas tributarias o aquella establecida por un informe de un especialista; del total de encuestados, nueve indican que aplicarían la depreciación en base al informe del especialista. De estos nueve encuestados, seis son mayores a 35 años y solo tres de ellos indican que aplican las NIIF. Siendo la mayoría de ellos hombres. 
En cuando a la preferencia en la utilización de las NIIF, en la elaboración de la información financiera, de los 50 encuestados, 27 respondieron que "preferir las normas contables sobre las tributarias en la elaboración de los EEFF", de los cuales solo son menores a 35 años, y solo siete de ellos aplica y conocen NIIF. Siendo cinco mujeres.

Solo 29 CPC consideran a las NIIF más importante que las normas tributarias en la elaboración de los estados financieros (pero dentro de ellos solo 12 indican conocen y aplican las NIIF), 12 de ellos considera que son menos importantes; 9 consideran que no son importantes.

Del total de encuestados, cuatro de ellos consideran que aprender las NIIF es fácil, 38 personas consideran que el nivel de dificultad es regular, 5 consideran que es difícil y 3 consideran que es muy difícil de aprender las NIIF.

Finalmente, sobre la pregunta: “QQué evento contable preferiría asistir?”, del total de encuestados, 26 de ellos asistirían a un curso sobre NIIF.

\section{Discusión}

En el presente estudio, observamos que la mayoría de encuestados, no conocen y ni utilizan las NIIF adecuadamente, debido a que el $66 \%$ de los contadores indican que no aplican las NIIF y que el $86 \%$ del total de encuestados respondió de forma incorrecta las preguntas de conocimiento. Por ello al no conocerlas no pueden aplicarlas adecuadamente en su información financiera con propósito general.
Pese a que el $58 \%$ de encuestados considera que son más importantes las NIIF que las normas tributarias en la elaboración de la información financiera, solo el $34 \%$ indica que las aplica y el $14 \%$ respondió de forma correcta el cuestionario de conocimientos básicos sobre NIIF. Esto demuestra que se conoce sobre la importancia de las NIIF, sin embargo se prefiere las normas tributarias en la elaboración de la información financiera.

Asimismo, los encuestados prefieren las normas tributarias en la aplicación de la información financiera, debido a que registran la información (depreciación tributaria en base a normas tributarias en el caso de leasing), y se capacitaron muy poco en temas relacionados a NIIF en los años 2013, 2012 y 2011; por lo que se infiere que no hay una capacitación constante en el tema, a pesar de que el $52 \%$ indica que desea asistir a eventos académicos relacionado con las NIIF en lugar de los de carácter tributario.

Una explicación al escaso conocimiento de las NIIF radica en su complejidad. Debido a ello, la SMV ha modificado la presentación de la información financiera auditada bajo NIIF en diciembre del 2014, a través de la resolución № 028-2014-SMV/o1. Por ello, para el 2015 presentarán la información auditada del 2014 aquellas entidades cuyos ingresos por ventas o prestación de servicios o con activos totales que al cierre del ejercicio 2014 sean iguales o superiores a 10,00o UITs, para el 2016 5,00o UITs y para el 2017 3,00o UITs, respectivamente.

En razón de todo lo expuesto, es necesario que los contadores se sigan capacitando en las NIIF, debido a que todo apunta a que 
todas de empresas utilicen las NIIF en la elaboración de información financiera.

Finalmente, debido a que la investigación fue de corte transversal y se realizó a principios de junio del año 2014, es posible que cambie la percepción de los contadores sobre las NIIF, ya que el Colegio de Contadores de Arequipa se encuentra realizando con más frecuencia eventos contables sobre NIIF, resaltando la importancia de su utilización en la elaboración de la información financiera. Sin embargo, se debe insistir en la importancia del conocimiento y la aplicación de las NIIF de manera adecuada y actualizada, según las últimas innovaciones en materia de finanzas y contabilidad.

\section{Referencias}

Alvarado, J. (2012). "Manual de Contabilidad Gubernamental”. 2da edición. Lima: Ediciones Gubernamentales.

Arroyo, A. (2011). Normas Internacionales de Información Financiera y el Peritaje Contable. "Contabilidad y Negocios", (6)12, 2011, 67-81.

Díaz, O. (2010). Estado actual de la aplicación de las NIIF en la preparación de estados financieros de las empresas peruanas. "Contabilidad y Negocios" (5)10, 5-28.

Díaz, O, A.; Durán, R. \& Valencia, A. (2012). Análisis de las diferencias entre el tratamiento contable y el fiscal para los elementos de propiedades, planta y equipo: el caso peruano. "Contabilidad y Negocios”, (5)10, 2010, 5-28.
Díaz, M.; Gil, J. \& Vilchez, P. (2010). Hacia la convergencia mundial del marco conceptual para la preparación de los estados financieros. "Contabilidad y Negocios” (5)9, 19-56.

Ernst \& Young. (2014). "Perú: EY abre oficina en Arequipa ante fuerte crecimiento de la región sur". Recuperado el o2 de junio del 2014 de http://www.ey.com/ PE/es/Newsroom/News-releases/ News-EY-abre-oficina-en-Arequipa

Hirache, L. \& Gonzales, E. (2011). "Nuevo Plan Contable General Empresarial”. Lima: Instituto Pacífico SAC.

International Accounting Standards Board. (2014). "Reino Unido: About the IFRS Foundation and the IASB". Recuperado o2 de junio del 2014 de http://www.ifrs. org/The-organisation/Pages/IFRSFoundation-and-the-IASB.aspx

Lam, A. (2010). La aplicación del nuevo Plan Contable General Empresarial. “Contabilidad y Negocios", (5)9, 5-18.

Mantilla, S. (2013). "Estándares / Normas Internacionales de Información Financiera IFRS (NIIF)”. 4ra Edición. Bogotá: Eco Ediciones.

Ministerio de Economía y Finanzas (2014). "Marco Macroeconómico Multianual Revisado 2014-2016". Recuperado el 25 de abril del 2014 de http://www.mef. gob.pe/contenidos/pol_econ/marco_ macro/MMM2014_2016_Rev.pdf

Palacios, M. \& Martínez, I. (2005). El proceso de armonización contable 
en Latinoamérica: camino hacia las normas internacionales. "Revista Contabilidad \& Finanzas", 16(39), 103-117.

Saavedra, E. (2012). "NIIFs Normas Internaciones de Información Financiera de Contabilidad. Teoría y práctica”. Lima: Ediciones Saavedra, Tarmeño \& Asociados SAC.

Valle, C. (2013). Globalización y contabilidad: Algunas reflexiones en torno a la obra El trabajo de las naciones de Robert B. Reich. "Contabilidad y Negocios”, (8)15, 95-101.

Zans, W. (2009). "Estados Financieros". Lima: San Marcos EIRL.

Zans, W. (2010). "Contabilidad Básica I". Lima: San Marcos EIRL.

Zans, W. (2012). "Plan Contable General Empresarial". 3ra edición. Lima: San Marcos EIRL. 